\title{
The Long-Term Study of Urinary Biomarkers of Renal Injury in Spontaneously Hypertensive Rats
}

\author{
Sebastián Montoro-Molina ${ }^{a}$ Andrés Quesada $^{b}$ Francisco O'Valle ${ }^{c}$ \\ Natividad Martín Morales ${ }^{c}$ María del Carmen de Graciab \\ Isabel Rodríguez-Gómez ${ }^{\mathrm{a}}$ Antonio Osuna ${ }^{\mathrm{b}, \mathrm{d}}$ Rosemary Wangensteen ${ }^{\mathrm{e}}$ \\ Félix Vargas ${ }^{\mathrm{a}, \mathrm{d}}$
}

aDepartamento de Fisiología, Facultad de Medicina, Granada, Spain; 'bServicio de Nefrología, Unidad Experimental, Hospital Virgen de las Nieves, Granada, Spain; 'Departamento de Anatomía Patológica e Instituto de Biomedicina Regenerativa (IBIMER), Facultad de Medicina, Granada, Spain; 'Instituto de Investigación Biosanitaria GRANADA,

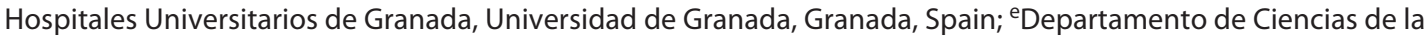
Salud, Universidad de Jaén, Jaén, Spain

\section{Keywords}

Spontaneously hypertensive rats · Aminopeptidases ·

Klotho - Dipeptidyl peptidase-4 - Biomarkers · Renal injury

\begin{abstract}
Background: The age-related increase in blood pressure in spontaneously hypertensive rats (SHRs) is associated to cardiac hypertrophy, heart failure, and renal injury. Here, we investigated for the first time the urinary enzymatic activities of glutamil aminopeptidase (GluAp), alanyl aminopeptidase (AlaAp), dipeptidyl peptidase-4 (DPP4), and Klotho urinary levels, proteins that are strongly expressed in the kidney, as early biomarkers of renal injury in SHRs. Methods: Male SHR and Wistar Kyoto (WKY) rats were studied from 2 to 8 months old. Systolic blood pressure (SBP), the heart rate (HR), metabolic variables, and urinary markers were measured monthly. At the end of the study, a histopathological evaluation of the kidney was performed. Results: Kidneys of SHR did not
\end{abstract}

develop signs of relevant histopathological changes, but showed increased glomerular area and cellularity. Plasma creatinine was decreased, and creatinine clearance was augmented in SHR at the end of the study. Urinary excretion of Klotho was higher in SHR at 5 and 8 months old, whereas plasma Klotho levels were similar to WKY. GluAp, AlaAp, and DPP4 urinary activities were increased in SHR throughout the time-course study. A positive correlation between glomerular area and cellularity with creatinine clearance was observed. Urinary GluAp, AlaAp, DPP4, and Klotho showed positive correlations with SBP. Conclusions: GluAp, AlaAp, DPP4, and Klotho in the urine are useful tools for the evaluation of renal damage at early stages, before the whole histopathological and biochemical manifestations of renal disease are established. Moreover, these observations may represent a novel and noninvasive diagnostic approach to assess the evolution of kidney function in hypertension and other chronic diseases.

(c) 2021 The Author(s)

Published by S. Karger AG, Basel karger@karger.com www.karger.com/kbr

Karger $\stackrel{\text { ' }}{5}$

BOPEN ACCESS
(C) 2021 The Author(s)

Published by S. Karger AG, Basel

This is an Open Access article licensed under the Creative Commons Attribution-NonCommercial-4.0 International License (CC BY-NC) (http://www.karger.com/Services/OpenAccessLicense), applicable to the online version of the article only. Usage and distribution for commercial purposes requires written permission. 


\section{Introduction}

The spontaneously hypertensive rat (SHR) is an experimental model of genetic hypertension $[1,2]$ that mimics human essential hypertension [2]. In this model of hypertension, blood pressure increases with age associated to renal injury, cardiac hypertrophy and heart failure [2]. Clinical nephrology claims for highly sensitive and specific diagnostic and prognostic biomarkers for acute and chronic kidney damage. The early detection of renal disease and the analysis of its progression are important objectives to improve the time course of renal injury.

Injured tubular cells excrete enzymes and other proteins to the urine that are potential biomarkers for an early detection of renal disease [3]. These cells contain aminopeptidases, mainly alanyl aminopeptidase (AlaAp) and glutamil aminopeptidase (GluAp), that have been described as early and predictive urinary biomarkers of renal injury severity $[4,5]$ and as an index of renal damage in hypertensive hyperthyroid rats [6]. Moreover, we also have reported that urinary Klotho levels are increased in Zucker obese rats and that they correlate with morphologic signs of renal injury and fibrosis in this experimental model [7].

Klotho is a transmembrane protein mainly expressed in tubular epithelial cells of several parts of the kidney [8]. There is a soluble extracellular domain, $\alpha$-Klotho, that is, cleaved from renal cells and released into the plasma [9]. Thus, Klotho can be found in 2 forms: tisular Klotho and released $\alpha$-Klotho. Soluble $\alpha$-Klotho is the functional form in the circulation, and it can cross from the blood renal tubules through transcytosis to the urine [10]. It has been reported that renal Klotho protein expression [1113] and plasma levels of Klotho are decreased in SHRs compared with Wistar Kyoto (WKY) rats [11]. Klotho gene activation in SHRs improved the course of hypertension and renal damage, abolished the upregulation of endothelin-1 levels, and increased the Mn-SOD expression in the kidney [14]. Moreover, the protective effects of angiotensin-I blockers in SHR seem to be mediated by upregulation of renal Klotho [12, 13]. For these reasons, Klotho has been proposed as a biomarker for renal and cardiovascular diseases $[15,16]$.

Dipeptidyl peptidase-4 (DPP4; EC no. 3.4.14.5) also called CD26, is a serine protease membrane glycoprotein with exopeptidase activity [17] DPP4 is expressed on the surface of several cell types, including epithelial, endothelial cells, and lymphocytes $[17,18]$. This peptidase also exists as a soluble circulating form (sDPP4) in the plasma and other body fluids [19]. A strong expression of DPP4 has been observed in the kidney [18], predominantly in the glomeruli and S1-S3 segments of the nephron [20], as well as in vascular endothelial cells suggesting that this protein may play a role in renal and cardiovascular function [21]. DPP4 seems to play an important role in diabetes, hypertension, and chronic kidney disease progression [20]. Variations in the concentration and activity of sDPP4 in the plasma have been widely used as a biomarker of many diseases. But, despite the evident linking of DPP4 with renal function, only a few studies have investigated sDPP4 activity in the context of hypertension.

The aim of this work was to investigate the plasma, urinary, and renal enzymatic activities of AlaAp, GluAp, and DPP4; and plasma and urinary Klotho levels as potential biomarkers of the renal injury and cardiovascular function in SHR for the first time. These proteins are strongly expressed in the kidney and have been revealed as better tools for early diagnostic, than the classic and other more recent biomarkers of renal disease [4]. To accomplish it, we performed a time-course study of these variables in the urine from 2 to 8 months of life. Finally, these variables were analyzed at the end of the experimental period in the plasma and renal tissue, and we also characterized the histopathological manifestations of renal injury in SHR.

\section{Methods}

\section{Animals}

Male SHR and WKY rats ( $n=10$, each group) of 2 months old were purchased from Harlan Laboratories (Barcelona, Spain) and studied up to 8 months old. These rats were kept in a room maintained at $24 \pm 1{ }^{\circ} \mathrm{C}$ and humidity of $55 \pm 10 \%$, with a 12-h light/dark cycle and had free access to rat chow $(24.3 \%$ of protein and $0.3 \%$ of sodium) and tap water.

\section{Experimental Protocol}

Tail systolic blood pressure (SBP) and heart rate (HR) were recorded monthly by using tail-cuff plethysmography in unanesthetized rats (LE 5001-pressure meter, Letica SA, Barcelona, Spain) from 2 to 8 months old. Each animal was placed in a heater at $37^{\circ} \mathrm{C}$ for $10 \mathrm{~min}$ and placed in a warm electric carpet to maintain vasodilation during determinations. SBP and HR were measured at least 10 times per rat waiting for a period of 2-3 min between each measurement. The mean value of the last 3 determinations that achieved a difference $<5 \mathrm{~mm} \mathrm{Hg}$ was taken as SBP and HR for each animal. All rats were weighed and housed monthly in metabolic cages (Panlab, Barcelona, and Spain) with free access to food and drinking water for a 24 -h period during which food and fluid intakes were measured and 24-h urine samples collected. Urine samples were centrifuged for $15 \mathrm{~min}$ at $1,000 \mathrm{~g}$ and frozen at $-80^{\circ} \mathrm{C}$ until assay. We measured monthly urine volume, proteinuria, AlaAp, GluAp, and DPP4 urinary activities. Urinary excretion of Klotho was measured at 2, 5, and 8 months old. These variables were expressed per $\mathrm{mg}$ of urinary creatinine. 
After completion of the experimental period (8 months old), rats were anaesthetized with ketamine $(80 \mathrm{mg} / \mathrm{kg}) /$ xylazine $(15$ $\mathrm{mg} / \mathrm{kg}$ ) i.p. Blood samples were then drawn by abdominal aortic puncture to determine plasma variables. Blood samples were centrifuged for $15 \mathrm{~min}$ at $1,000 \mathrm{~g}$ and stored at $-80^{\circ}$. Finally, the rats were killed by cervical dislocation and kidneys and heart were quickly removed and weighed. Plasma variables were: urea, creatinine, AlaAp, GluAp, DPP4, and Klotho. The tibial length was measured to normalize the heart and kidney weight, since body weight cannot be used for this purpose in the present experimental setting. One kidney was harvested without perfusion, fixed in $10 \%$ neutral buffered formaldehyde solution for $48 \mathrm{~h}$ and subsequently placed in $70 \%$ ethanol for histological studies.

\section{Analytical Procedures}

Proteinuria and urine creatinine were determined in urine samples by an autoanalyzer Spin120. Plasma creatinine and urea were also measured in this instrument. Reagents for proteinuria (pyrogallol red method), urea (o-phthalaldehyde method), and creatinine (Jaffé method) were provided by Spinreact (Barcelona, Spain). Plasma and urinary Klotho levels were determined with an enzyme immunoassay kit, Bioassay Technology Laboratory (Shangai, China).

\section{Aminopeptidasic Activities Measurement}

GluAp, AlaAp, and DPP4 activities were determined in duplicate in a fluorimetric assay using glutamyl $\beta$-naphthylamide, alanyl $\beta$-naphthylamide, and glycil-prolyl 4 -methoxi- $\beta$-naphthylamide as substrates. In brief, $20 \mu \mathrm{L}$ of the urine or plasma were incubated for $30 \mathrm{~min}$ at $37^{\circ} \mathrm{C}$ with $80 \mu \mathrm{L}$ of their corresponding substrate solution (10 mM in HCl-Tris $50 \mathrm{~mm} \mathrm{pH} \mathrm{8.7).} \mathrm{The} \mathrm{amount}$ of $\beta$-naphthylamine or 4 -methoxi- $\beta$-naphthylamine released as a result of aminopeptidasic activities was measured fluorimetrically each minute at an emission wavelength of $412 \mathrm{~nm}$, with an excitation wavelength of $345 \mathrm{~nm}$, and quantified using a standard curve for $\beta$-naphthylamine or 4 -methoxi- $\beta$-naphthylamine containing $0-200 \mathrm{nmol} / \mathrm{mL}$. Specific activities were calculated from the slope of the linear portion of enzymatic assay, and expressed as nanomol of substrate hydrolyzed per $\mathrm{mL}$ and minute.

\section{Histopathological Study}

For conventional morphology, buffered $10 \%$ formaldehydefixed, paraffin-embedded longitudinal rat kidney sections in the sagittal plane were deparaffinized in xylol ( 3 passes of $5 \mathrm{~min}$ ) and rehydrated in ethanol of decreasing gradation (absolute, 96 and $70 \%, 2$ passes of $3 \mathrm{~min}$, respectively). Tissue sections were stained with Masson's trichrome. The presence of glomerular lesions (glomerulosclerosis glomerular hyperplasia, mesangium increase, glomerular cyst, and capsular fibrosis) was assessed in at least 200 glomeruli. Tubulointerstitial damage (tubular vacuolation, tubular atrophy, hyaline drops, tubular casts, and chronic inflammatory infiltrate) was also studied. Injury was graded according to Shih et al. [22] on a semiquantitative scale of $0-4(0=$ normal, $0.5=$ small focal areas of damage, $1=$ involvement of $<10 \%$ of the cortex, 2 = involvement of $10-25 \%$ of the cortex, 3 = involvement of $25-75 \%$ of the cortex, and $4=$ extensive damage involving $>75 \%$ of the cortex) [22]. The morphological study was done in blinded fashion on $4 \mu \mathrm{m}$ sections with light microscopy.

\section{Morphometrical Study}

Samples were fixed in buffered $10 \%$ formalin, embedded in paraffin, and serially sectioned at $5 \mu \mathrm{m}$ thickness. Afterwards, they were stained with $1 \%$ picrosirius red F3BA (Gurr, BDH Chemicals Ltd., Poole, UK) for image analysis quantification. To improve staining, tissue sections were kept after deparaffination for 3-5 days in $70 \%$ alcohol as mordent. Picrosirius red stains connective fibers deep red and cell nuclei and cytoplasmatic structures light red/ bright yellow [23]. To semiautomatically quantify interstitial connective tissue, 20 images of cortex and corticomedullary junction per kidney were acquired using a digital camera 3CCD (DP70) coupled to an Olympus BX-42 brightfield microscope (Olympus Optical Co., Ltd) equipped with polarizing crystals. Twenty images of corticomedullary junction per kidney were acquired using polarized light. Histologic images of kidney biopsies were acquired using polarized light and were converted to black and white at 8-bit intensity resolution (256 gray levels) with a global magnification of $\times 200$, and normalized with Adobe Photoshop software (Adobe Systems Software, Ireland). To assess the fibrosis, we made a macro that included a semiautomatic thresholding of the total of the images per group of rats simultaneously with ImageJ software (v 1.48) (National Institute of Health, USA. http://imagej.nih.gov/ij/).

\section{Statistical Analyses}

To study the time course of biological variables and urinary markers, we used a factorial: ANOVA for repeated measures, taking each rat as the subject and the group (WKY or SHR) as the between-subjects factor. Interactions between factors were analyzed using the Bonferroni method. We used Statgraphics Centurion XVIII software.

Morphological and biological variables measured at the end of the experiment were compared using a $t$ test for the analysis of variables with normal distribution and equal variances. Welch modification of the $t$ test was used for data with normal distribution and unequal variances. The Shapiro-Wilk test was used to analyze the normality of distributions. Differences were considered statistically significant at $p<0.05$ level.

Simple linear regressions were analyzed with Statgraphics Centurion XVII software including all data in a common regression line. For histopathological results, IBM SPSS-Windows 20.0 (SPSS Inc., Chicago, IL, USA) was used for the analyses. Results are presented as mean \pm standard error. Results were considered statistically significant when $p$ values were below 0.05 .

\section{Results}

\section{Time Course of Biological Variables}

Figures 1 and 2 summarize the comparison of the time course of biological variables between SHR and WKY rats. The variables studied were: SBP, HR, body weight, food intake, water intake, diuresis, and water balance. SBP was obviously greater in SHR with respect to WKY rats, and showed a time-related increase (Fig. 1A). HR showed significant increases at months 6,7 , and 8 (Fig. 1B). The time-related body weight increase was significantly reduced in SHR from the 4th month until the 


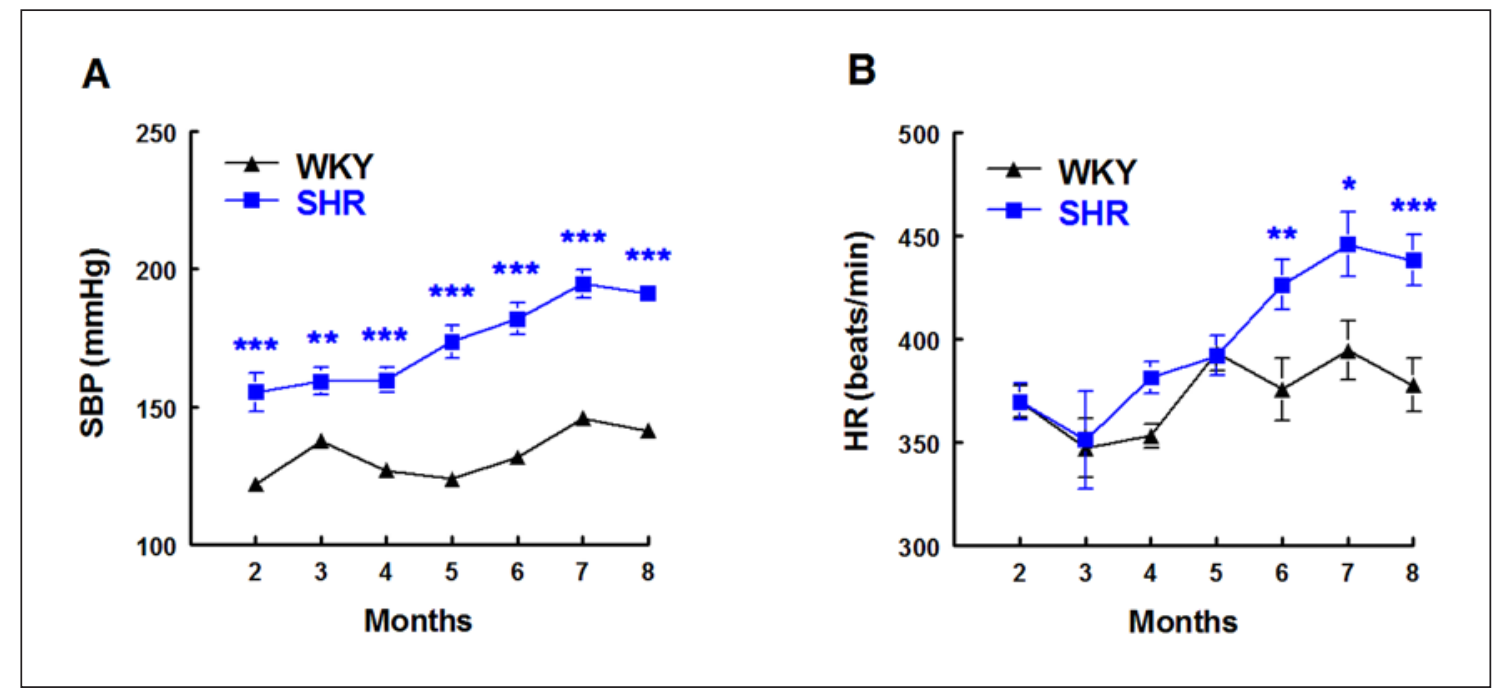

Fig. 1. Time course of tail SBP $(\mathbf{A})$ and HR $(\mathbf{B})$ during the experimental period. Data are means \pm SEM. ${ }^{*} p<0.05$, ${ }^{* *} p<0.01$, and ${ }^{* * *} p<0.001$ compared with WKY rats $(n=10$, each group). WKY, Wistar Kyoto; SBP, systolic blood pressure; HR, heart rate.

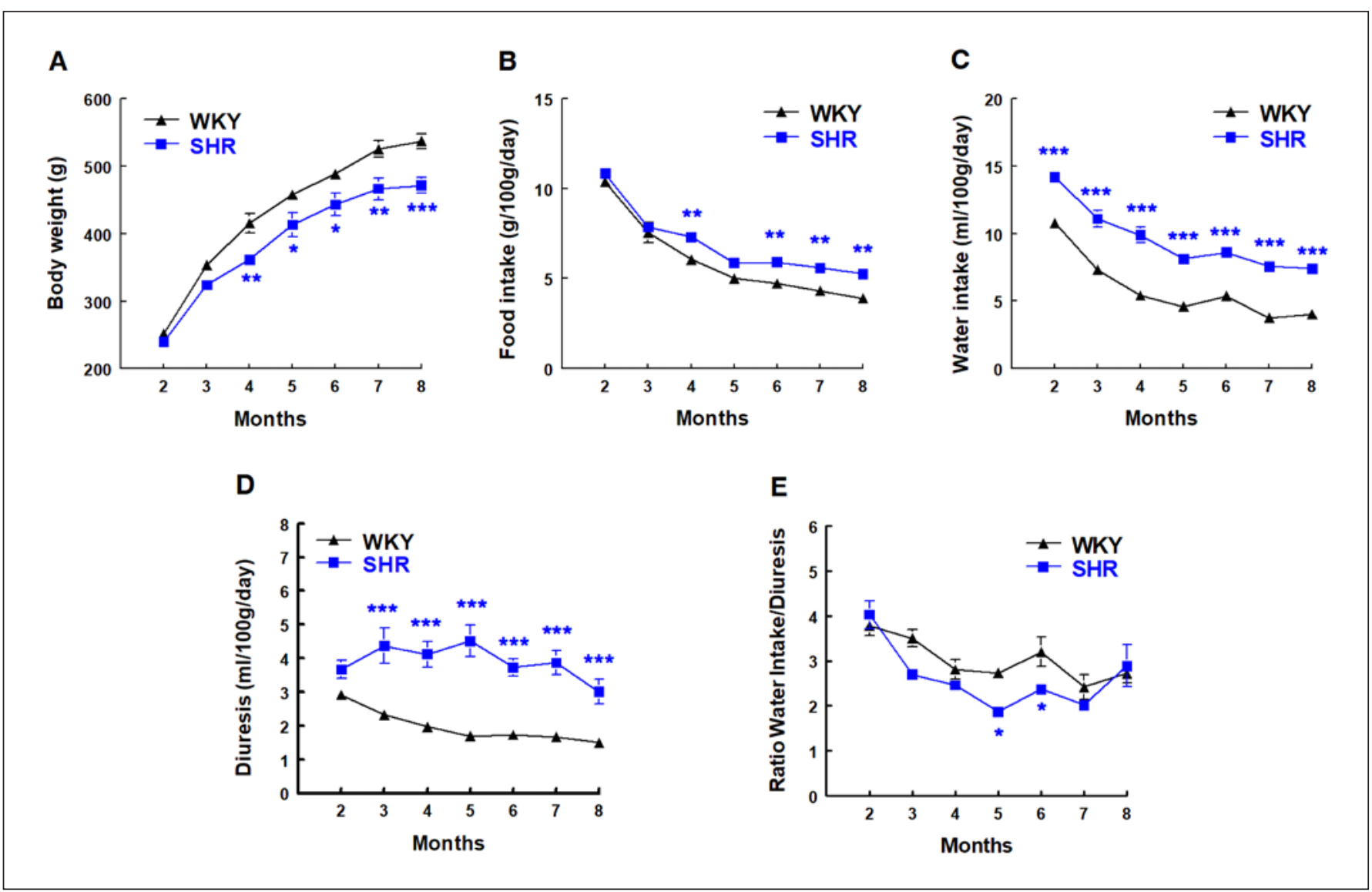

Fig. 2. Time course of biological variables. Body weight (A), food intake (B), water intake (C), diuresis (D), and ratio of water intake/diuresis $(\mathbf{E})$. Data are means \pm SEM. ${ }^{*} p<0.05^{* *} p<0.01$, and ${ }^{* * *} p<0.001$ compared with WKY rats ( $n=10$, each group). WKY, Wistar Kyoto. 
Fig. 3. Representative microphotograph of renal cortex in WKY (A) and SHR (B) displaying the absence of tubulointerstitial or vascular injury. Bar: $100 \mu \mathrm{m}$ (Masson's Trichome stain, original magnification $\times 4$ ). Increase in the glomerular size and cellularity in SHR (D) compared with WKY rats (C). Bar: $50 \mu \mathrm{m}$ (Masson's Trichome stain, original magnification $\times 40$ ). Changes in glomerular area $(\mathbf{E})$ and cellularity $(\mathbf{F})$ in the glomerulus of SHR and WKY rats $(n=$ 20 glomeruli per rat). Data are means \pm SEM. ${ }^{*} p<0.05,{ }^{* *} p<0.001$ compared with WKY rats ( $n=10$, each group). SHR, spontaneously hypertensive rat; WKY, Wistar Kyoto.

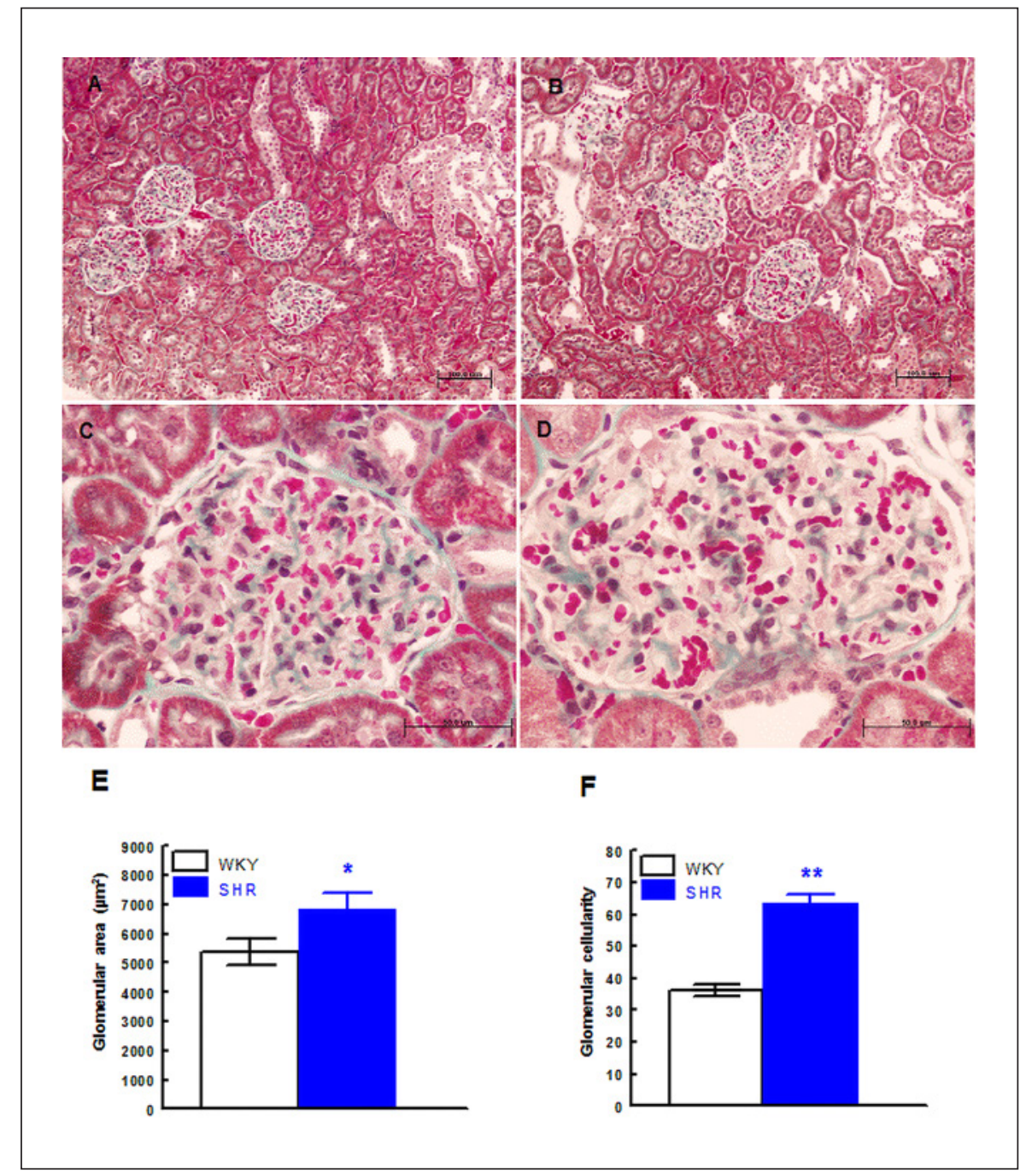

Table 1. Morphological variables in WKY and SHR at 8 months old

\begin{tabular}{lcc}
\hline Group & WKY & SHR \\
\hline Body weight (BW, g) & $537 \pm 11.1$ & $472 \pm 11.6^{* * *}$ \\
Tibial length (TL, cm) & $5.79 \pm 0.04$ & $5.57 \pm 0.05^{* *}$ \\
Kidney weight (KW, g) & $1.42 \pm 0.03$ & $1.38 \pm 0.05$ \\
KW/TL (mg/cm) & $245 \pm 5.22$ & $247 \pm 8.09$ \\
KW/BW (mg/g) & $2.64 \pm 0.04$ & $2.92 \pm 0.09^{*}$ \\
Heart weight (HW, g) & $1.20 \pm 0.02$ & $1.52 \pm 0.04^{* * *}$ \\
HW/TL (mg/cm) & $206 \pm 3.05$ & $273 \pm 4.74^{* * *}$ \\
HW/BW (mg/g) & $2.23 \pm 0.03$ & $3.23 \pm 0.08^{* * *}$ \\
\hline
\end{tabular}

Data are expressed as means \pm SE. BW, Final body weight; TL, tibial length; KW, kidney weight; KW/TL, ratio kidney weight/ tibial length; KW/BW, ratio kidney weight/body weight; HW, heart weight; $\mathrm{HW} / \mathrm{TL}$, ratio heart weight/tibial length. ${ }^{*} p<0.05$, ${ }^{* *} p<0.01,{ }^{* * *} p<0.001$ vs. WKY group ( $n=10$ in each group). end of the study with respect to WKY rats. Food intake relative to body weight was time-related decreased in both SHR and WKY. This variable was significantly greater in SHR at 4, 6, 7, and 8 months. Water intake relative to body weight also was time-related decreased in both SHR and WKY, being significantly higher in the SHR group. In consonance, diuresis relative to body weight also was increased in SHR along the study. The ratio of water intake/diuresis was reduced at 5 th and 6 th months in SHR with respect to WKY rats. These results are included in Figure 2A-E.

\section{Morphological Variables}

Final body weight was significantly reduced in SHR when compared to WKY rats. Kidney weight in absolute value or relative to tibial length was similar in SHR and 
Table 2. Plamatic and renal variables in WKY and SHR at 8 months old

\begin{tabular}{lcc}
\hline Group & WKY & SHR \\
\hline Urea $(\mathrm{mg} / \mathrm{dL})$ & $70.7 \pm 0.36$ & $69.8 \pm 0.40$ \\
Creatinine $(\mathrm{mg} / \mathrm{dL})$ & $0.478 \pm 0.018$ & $0.359 \pm 0.013^{* * *}$ \\
Creatinine clearance $(\mathrm{mL} / \mathrm{min})$ & $1.297 \pm 0.061$ & $1.756 \pm 0.152^{*}$ \\
Creatinine clearance $(\mathrm{mL} / \mathrm{min} / 100 \mathrm{~g})$ & $0.241 \pm 0.009$ & $0.372 \pm 0.031^{* *}$ \\
Creatinine clearance $(\mathrm{mL} / \mathrm{min} / \mathrm{g}$ kidney) & $0.457 \pm 0.015$ & $0.636 \pm 0.048^{* *}$ \\
\hline
\end{tabular}

Data are expressed as means \pm SE. ${ }^{*} p<0.05,{ }^{* *} p<0.01,{ }^{* * *} p<0.001$ vs. WKY group ( $n=10$ in each group).
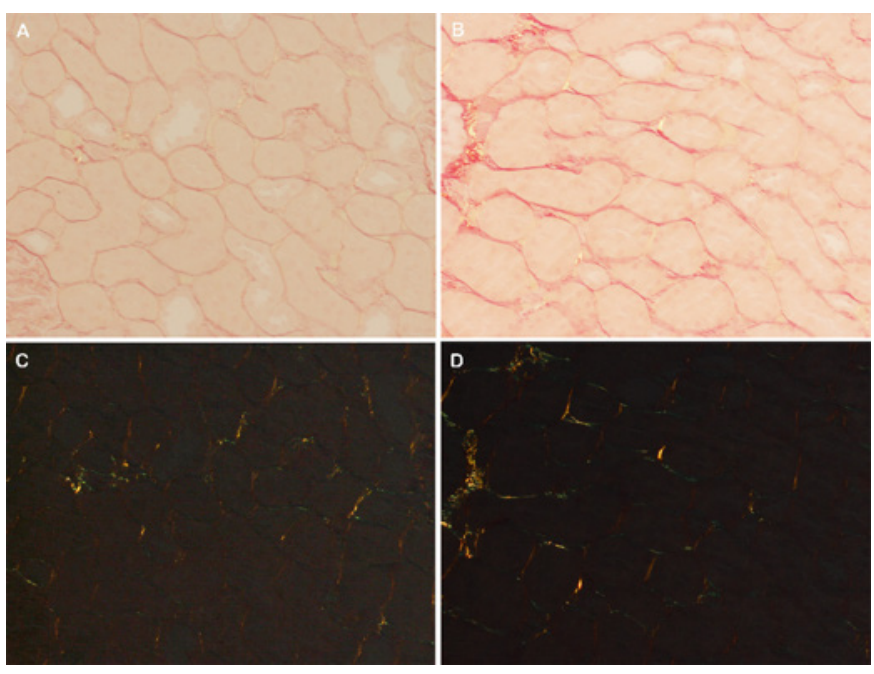

Fig. 4. Representative microphotograph of renal cortex and corticomedullary junction in WKY $(\mathbf{A}, \mathbf{C})$ and SHR $(\mathbf{B}, \mathbf{D})$ displaying the area of interstitial connective tissue obtained with picrosirius red stain using nonpolarized (up) and polarized (down) light. Twenty images per kidney were quantified. SHR, spontaneously hypertensive rat; WKY, Wistar Kyoto.

WKY rats, but was increased in SHR relative to body weight. Heart weight was significantly increased in SHRs regardless of the way of expression, when compared with the WKY group (Table 1).

\section{Histopathological Results}

Renal lesions in the SHR group were absent and no glomerular, tubulointerstitial, or vascular lesions were present in renal parenchyma in SHR, as seen in Figure 3A-D. However, the SHR group showed a significant increase in glomerular area and glomerular cellularity with respect to WKY rats (Fig. 3E, F). We did not find any significant difference in the percentage of area of interstitial connective tissue determined by sirius red staining in WKY $(1.77 \pm$ $0.15)$ and SHR (2.53 \pm 0.38$)$ groups (Fig. 4A-D).

\section{Plasma Variables and Creatinine Clearance at the} End of the Study

Plasma urea was similar in SHR and WKY rats, but plasma creatinine was decreased in SHR. Creatinine clearance $(\mathrm{CrCl})$ was augmented in SHR expressed in absolute values or relative to body weight or kidney weight. These data are shown in Table 2.

\section{Time Course of Urinary Klotho and Plasma \\ Concentration}

Urinary excretion of Klotho (ng/mg creatinine) was higher in SHR at 5 and 8 months old (Fig. 5E). Plasma levels of Klotho (Table 3) were similar in SHR and WKY rats at the end of the experimental period ( 8 months old).

\section{Time Course of Urinary Enzymatic Activities,}

Proteinuria, and Creatinine Excretion

Figure 5B-E shows the evolution of enzymatic urinary biomarkers along the study when data were normalized by creatinine excretion. GluAp was increased in SHR throughout the study, AlaAp was increased at 2, 4, 6, and 7 months old, and DPP 4 was increased from 4 to 8 months old. Proteinuria was increased at 2, 3, 6, and 7 months old. Urinary creatinine excretion was significantly increased at 4 and 5 months old in the SHR group (Fig. 5A).

\section{Aminopeptidasic Activities in the Plasma and Kidney} of WKY and SHR at the End of the Study

The plasma levels of GluAp and DPP4 activities were increased in SHR with respect to WKY rats, but AlaAp activity was significantly decreased in SHR. In the whole kidney, AlaAp and DPP4 were similar in SHR and WKY, but GluAp activity was decreased in SHR with respect to the control group. All these data are given in Table 3.

\section{Correlation Studies}

As urinary Klotho and urinary aminopeptidases have shown positive correlations in other experimental condi- 
Table 3. Aminopeptidasic activities and Klotho in plasma and kidney of WKY and SHR at 8 months old

\begin{tabular}{lll}
\hline Group & WKY & SHR \\
\hline Plasma & & \\
GluAp (nmol/mL/min) & $0.97 \pm 0.03$ & $1.54 \pm 0.05^{* * *}$ \\
AlaAp (nmol/mL/min) & $2.41 \pm 0.05$ & $2.13 \pm 0.05^{* *}$ \\
DPP4 (nmol/mL/min) & $2.74 \pm 0.05$ & $3.05 \pm 0.12^{*}$ \\
Klotho (ng/mL) & $4.30 \pm 0.16$ & $4.61 \pm 0.15$ \\
Kidney & $19.1 \pm 0.71$ & $15.7 \pm 1.11^{*}$ \\
GluAp (nmol/min/mg protein) & $22.5 \pm 0.84$ & $27.2 \pm 1.89$ \\
AlaAp (nmol/min/mg protein) & $46.1 \pm 1.81$ & $45.5 \pm 2.59$ \\
DPP4 (nmol/min/mg protein) & \\
\hline
\end{tabular}

Data are expressed as means \pm SE. ${ }^{*} p<0.05,{ }^{* *} p<0.01,{ }^{* * *} p<0.001$ vs. WKY group ( $n=10$ in each group).
A

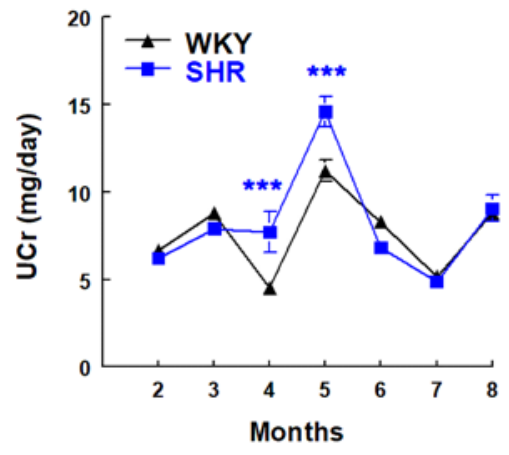

D

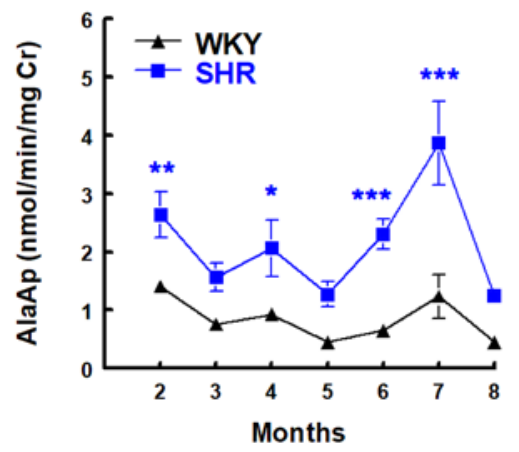

B

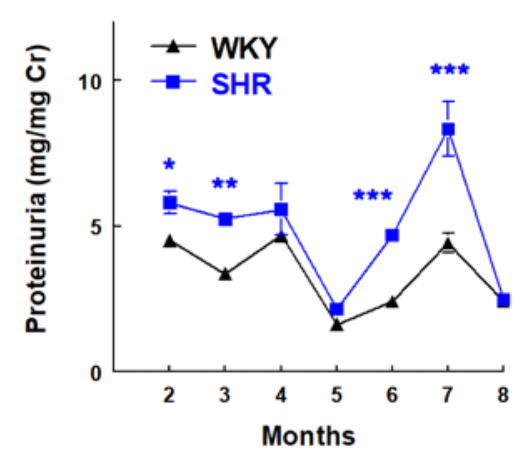

E

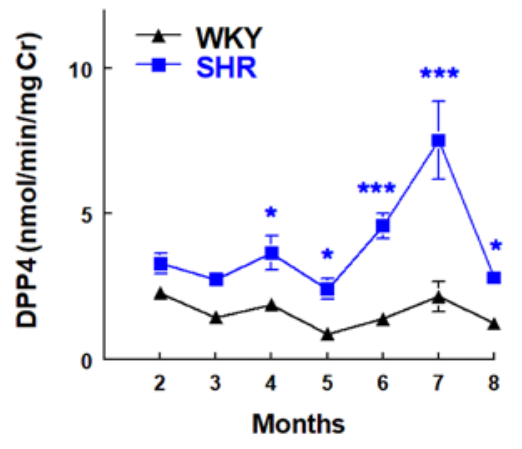

C

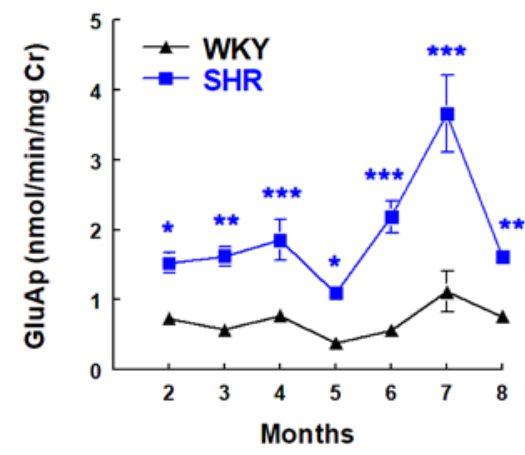

$\mathbf{F}$

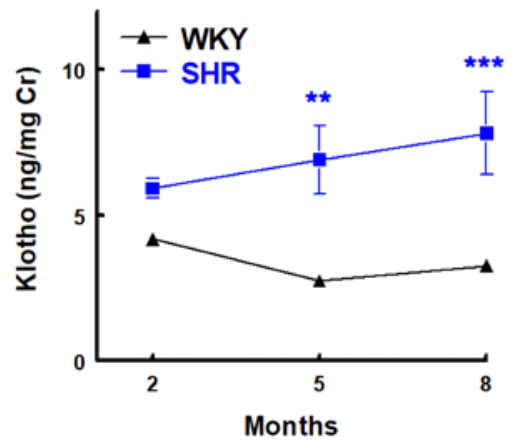

Fig. 5. Time course of renal injury biomarkers normalized by creatinine excretion. UCr (A), proteinuria (B), GluAp (C), AlaAp (D), and DPP4 (E) activities, and Klotho excretion (F) in male SHR and WKY rats. Data are means \pm SEM. ${ }^{*} p<0.05{ }^{* *} p<0.01$, and ${ }^{* * *} p<0.001$ compared with WKY rats $(n=10$, each group). GluAp, glutamil aminopeptidase; AlaAp, alanyl aminopeptidase; DPP4, dipeptidyl peptidase-4; SHR, spontaneously hypertensive rat; WKY, Wistar Kyoto; UCr, urinary creatinine. 
Fig. 6. Correlation between urinary Klotho and proteinuria $(\mathbf{A})$ or urinary aminopeptidasic activities (B, C, D) measured at 8 months old, when all animals (SHR and WKY rats) are pooled in a common regression line. SHR, spontaneously hypertensive rat; WKY, Wistar Kyoto.
A

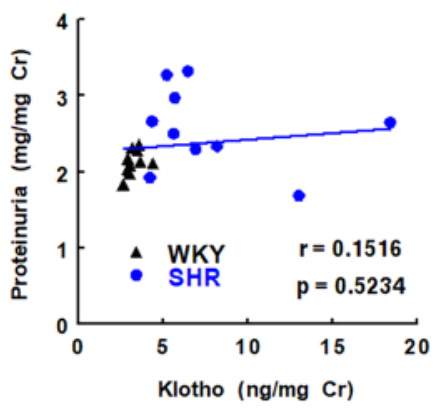

C

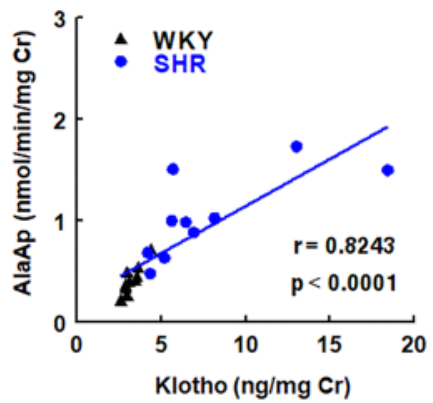

B

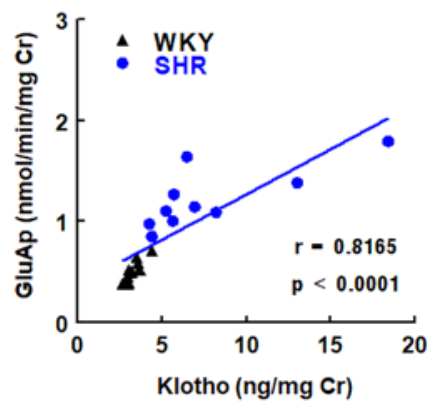

D

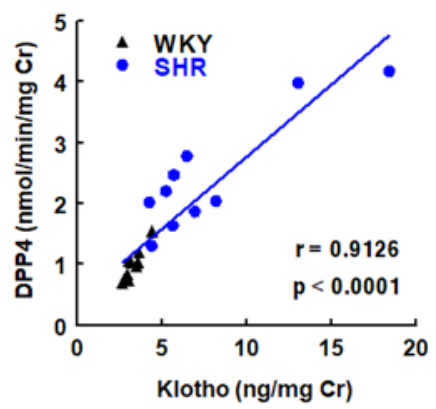

Fig. 7. Correlation between $\mathrm{CrCl}$ and glomerular cellularity $(\mathbf{A})$ or glomerular area (B) at the end of the study (8 months old), when all animals (SHR and WKY rats) are pooled in a common regression line. SHR, spontaneously hypertensive rat; WKY, Wistar Kyoto; $\mathrm{CrCl}$, creatinine clearance.
A

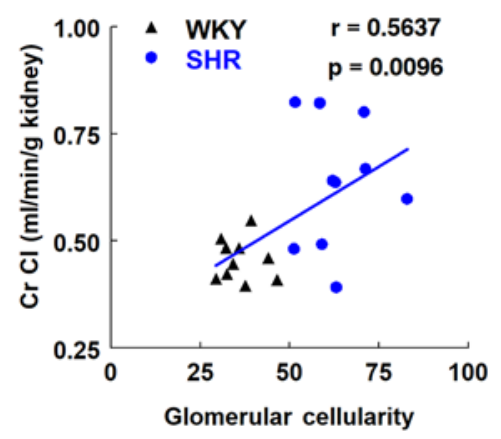

B

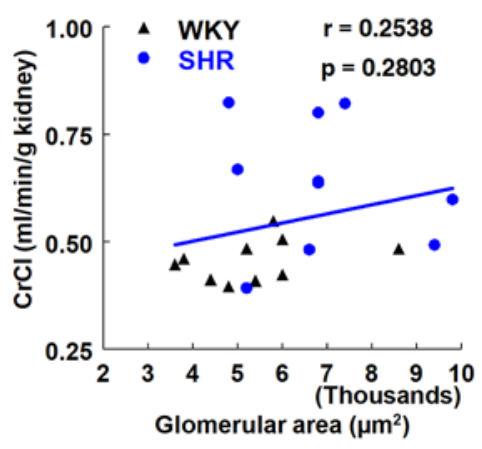

tions of renal injury, we decided to determine the relationship between these variables in SHR. Urinary Klotho levels showed positive correlations with urinary GluAp, urinary AlaAP, and urinary DPP4 excreted at 8 months old, when all animals (SHR and WKY rats) are pooled in a common regression line, reaching the strongest correlation with urinary DPP4 (Fig. 6).

To study the relationship between histopathological glomerular findings and $\mathrm{CrCl}$ changes in SHR, the correlation between $\mathrm{CrCl}$ with glomerular cellularity and area was analyzed. Positive correlations were found for these 2 variables with $\mathrm{CrCl}$, reaching a marked statistical signification between $\mathrm{CrCl}$ and glomerular cellularity (Fig. 7). Nevertheless, no correlation was found when data of SHR and WKY groups were separately analyzed (data not shown).

In order to evaluate the interaction between the evolution of urinary biomarkers and time-related blood pressure changes observed in this hypertensive model, the correlations between the mean values of these variables at 


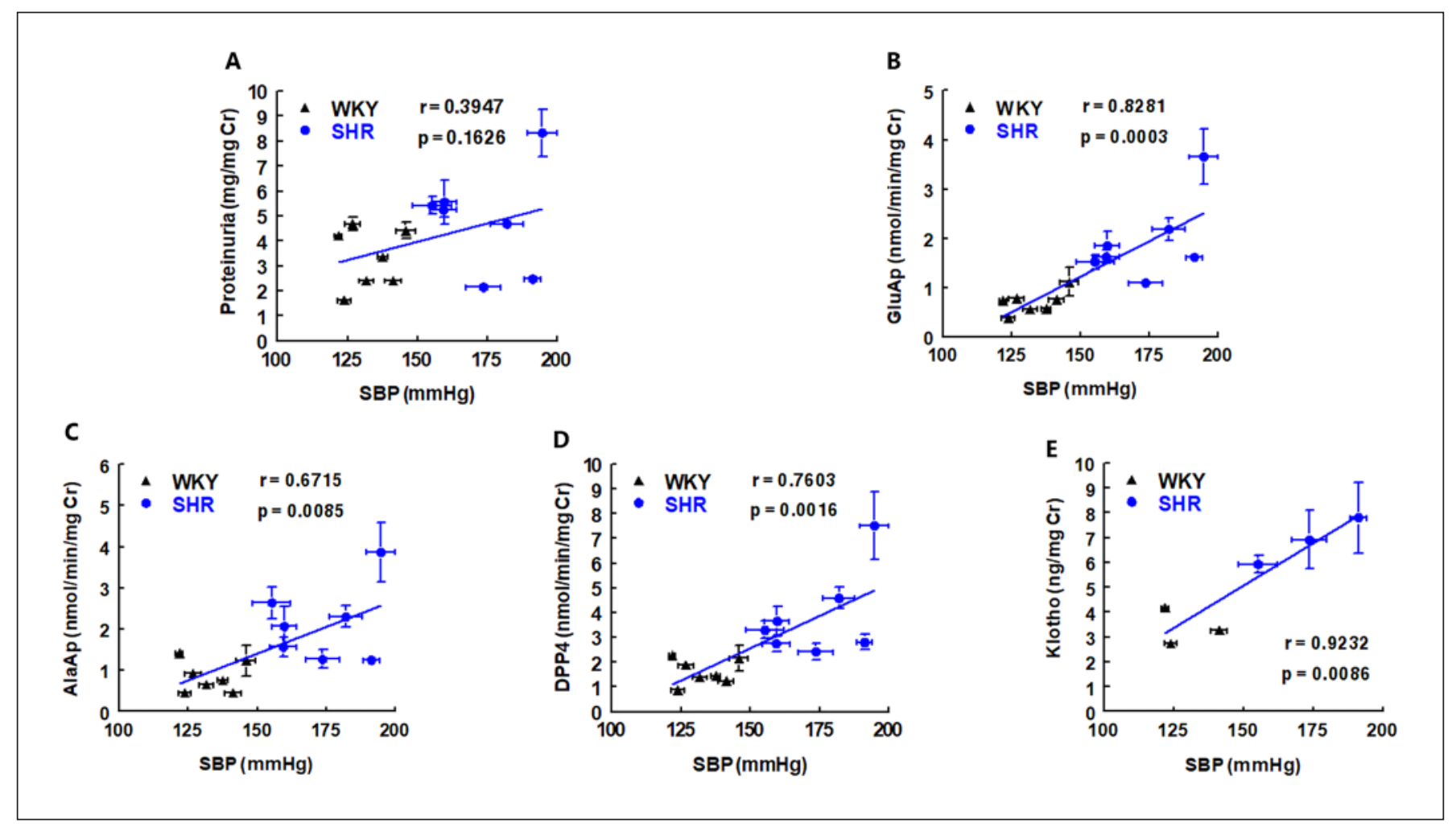

Fig. 8. Correlations between the time course of SBP and urinary biomarkers: proteinuria (A), GluAp (B), AlaAp (C), and DPP4 (D) activities and urinary Klotho (E) mean values at each month of the study, when all animals (SHR and WKY rats) are pooled in a common regression line. GluAp, glutamil aminopeptidase; AlaAp, alanyl aminopeptidase; DPP4, dipeptidyl peptidase-4; SHR, spontaneously hypertensive rat; WKY, Wistar Kyoto; SBP, systolic blood pressure.

each month of the study were analyzed. We found that all urinary biomarkers, except proteinuria, showed positive correlations along the study with the time course of SBP, when all data (WKY and SHR) were pooled in a common regression line, obtaining the strongest relationship with urinary Klotho $(r=0.9232$; $p<0.0086)$ (Fig. 8).

\section{Discussion}

This study clearly shows for the first time that spontaneous hypertension is associated to increased urinary GluAp, AlaAp, and DPP4 activities and urinary excretion of Klotho, changes that were detected as early as 2 months old in the case of GluAp and AlaAp. This study also shows that all these variables, but not proteinuria, strongly correlate with SBP.

Our data show that plasma creatinine is decreased and creatinine clearance was augmented in SHR at the end of the study (8 months old), probably due to hyperfiltration secondary to increased glomerular capillary pressure previously reported [24]. Our data also agree with the time course of this variable reported by Hultström [24], showing that the glomerular filtration rate is progressively increasing until 15 weeks old, then it reaches a plateau and it starts to decline at 60 weeks ( 15 months old). In our work, we found a higher urinary creatinine excretion in SHR rats at 4 and 5 months old, coinciding with the increased glomerular filtration rate described by Hultström at these time points [24] Moreover, our study also shows that SHR group displayed an augmented glomerular area and an increased cellularity in the glomerulus, which might contribute to hyperfiltration in SHR. In fact, in this study, a negative correlation is reported between glomerular cellularity and serum creatinine.

Histopathological results indicate that no glomerular, tubulointerstitial, or vascular lesions were present in renal parenchyma in SHR, in consonance with a previous report showing that the kidney in SHR is protected against renal injury by an increased resistance in the afferent ar- 
teriole [25]. Thus, it has been reported that hypertensive kidney damage is not morphologically evident before 7.5 months old $[26,27]$. Renal damage progresses with increasing age, and at 60 weeks old, SHR show widespread and quite severe lesions [28].

It is interesting to note that despite the absence of relevant histopathological findings of renal injury in SHR, the decreased levels of plasma creatinine and the increased creatinine clearance, all the urinary markers of tubular injury were increased in SHR. These data indicate that an incipient tubular injury might be present before renal disease is fully manifested, because the kidney has a high reserve capacity and tubular cells have a high regenerative capacity [29]. Therefore, the classic biomarkers of renal disease are not useful tools for the evaluation of hypertensive renal damage at this stage.

Proteinuria is considered as a marker of the progression of renal injury. SHR shows increased proteinuria that started to reach signification at the first determination ( 2 months old). In consonance with this, in male SHR it has been reported as increased proteinuria at a relatively younger age (6 weeks old) [30] that also agree with a previous report from our laboratory [31]. However, other authors reported the appearance of proteinuria in SHR in later stages, starting to increase at 28-30 weeks old [24]. SHR excrete a mild amount of urinary protein, despite high blood pressure, that contrast with the higher levels reported in volume dependent types of hypertension [32]. Consistent with the mild increase in urinary protein, histopathological data did not show severe morphologic changes in the renal tissues of SHR at 8 months old despite the high levels of blood pressure. In our study, proteinuria was very changeful at different time points, and there was not any correlation with SBP. It is also remarkable that proteinuria observed at 5 months old was increased in a parallel manner to urinary creatinine excretion in SHR. As a consequence, when proteinuria is normalized by creatinine, it is not increased at this point. This finding suggests that glomerular hyperfiltration is accompanied by proteinuria, at least at this time point, but it also demonstrates that quantification of urinary markers by creatinine excretion in a spot sample cannot be adequate in a disease or an animal model that course with glomerular hyperfiltration because of the high excretion of creatinine.

GluAP and AlaAP were increased in SHR along the study, and DPP4 reached statistical signification from 4 months old. The data agreed with previous articles, where we showed that early changes in the urinary activity of these enzymes were linked with the progression of renal disease in hypertensive hyperthyroid rats [6], in cisplatin treated rats $[4,5]$, and more recently in Zucker obese rats [7]. These enzymes serve to detect proximal tubular injury regardless of the glomerular filtration status [33] because they are released to the urine from damaged tubular cells [34]. It is interesting to note that, despite the absence of histopathological findings of renal injury, these urinary biomarkers are increased in SHR, indicating that biochemical abnormalities precede the detection of tubular morphological changes. Moreover, the urinary biomarkers analyzed along the study (GluAp, AlaAp, DPP4, and Klotho) showed positive correlations with SBP, suggesting that increased BP activated some degree of tubular injury not detectable by conventional histopathological evaluation.

This study shows increased urinary levels and normal plasma and renal levels of Klotho in SHR that contrast with the decreased plasma levels and renal Klotho protein expression previously reported in this hypertension model in rats of similar age $[12,13]$. This wasting of Klotho through the urine, might determine undesirable effects in SHR, since Klotho gene delivery has protective effects in the progression of hypertension and renal damage [14], and antihypertensive treatment is associated to upregulation of renal Klotho expression [12, 13]. The mechanism of wasting of Klotho through the urine is unknown, but the positive correlation between urinary aminopeptidases of the tubular origin and urinary Klotho, as previously reported in Zucker obese rats [7], indicates that the concomitant augment of these urinary variables may be due to a tubular dysfunction.

Plasma DPP4 activity is higher in SHRs compared to WKY rats, data that agree with those of Pacheco et al. [35] that also reported that this elevation precedes the onset of hypertension. This enzyme must play an important role in the pathogenesis of spontaneous hypertension, since the administration of sitagliptin, a specific DPP4 inhibitor, exerts important renal [35] and vascular [36] protective effects in SHRs. DPP4 also has been considered as a diagnostic or prognostic biomarker for several clinical events as diabetes [37], coronary artery disease [38], renal function, and cardiovascular risk [37, 39]. Moreover, high plasma levels of sDPP4 have been also associated to liver [40] and cardiac [41] fibrosis. Therefore, our plasma and urinary results together with all these observations suggest the potential use of urinary DPP4 levels as a future biomarker of decreased renal function and fibrosis.

In conclusion, the glomerular histopathological changes observed in SHR might participate in the hyper- 
filtration of this model. The fact that all urinary markers of tubular injury were increased in SHR might indicate that a subjacent renal injury can be detected before the whole histopathological and biochemical manifestations of renal disease are present. Therefore, the present urinary biomarkers of renal disease are useful tools for the evaluation of renal damage at early stages. Finally, the determination of the biomarkers analyzed in this study may serve as a noninvasive diagnostic approach to assess renal injury in hypertension and other chronic renal diseases.

\section{Acknowledgments}

Technical and human support provided by CICT of Universidad de Jaén (UJA, MINECO, Junta de Andalucía, FEDER) is gratefully acknowledged. We also thank the technical assessment of María Dolores Rodríguez.

\section{Statement of Ethics}

All experimental procedures were performed according to the European Union Guidelines to the Care and Use of Laboratory Animals and approved by the Ethical Committee of the University of Jaén and the Service of Animal Health of Government of Andalucía with the approval ID 450-5297 on February 21, 2014.

\section{Conflict of Interest Statement}

Rosemary Wangensteen, Félix Vargas, and Antonio Osuna are coauthors of the patent "Aminopeptidases as markers of renal damage" with publication number 2382960 . There is not any other relevant declaration related to employment, consultancy, patents, products in development, or modified products.

\section{Funding Sources}

This study was supported by the grants PI13/02743, PI13/02384, and PI18/01715 from the Carlos III Health Institute of Spain, and the Red de Investigación Renal REDinREN RD16/0009/0033. "FEDER una manera de hacer Europa."

\section{Author Contributions}

Sebastián Montoro-Molina, Andrés Quesada, Francisco O’Valle, Natividad Martín Morales, María del Carmen de Gracia, Isabel Rodríguez-Gómez; Antonio Osuna, Rosemary Wangensteen, and Félix Vargas conducted the experiments and prepared the materials involved in this study. Félix Vargas and Rosemary Wangensteen conceived this study. F. Vargas participated in its design and coordination. Félix Vargas, Sebastián Montoro-Molina, Francisco O'Valle, and Rosemary Wangensteen contributed to the analysis and interpretation of the data. Rosemary Wangensteen, Sebastián Montoro-Molina and Félix Vargas drafted the manuscript. All authors read and approved the final manuscript.

\section{References}

1 Llorens S, Fernández AP, Nava E. Cardiovascular and renal alterations on the nitric oxide pathway in spontaneous hypertension and ageing. Clin Hemorheol Microcirc. 2007;37: $149-56$.

2 Sun ZJ, Zhang ZE. Historic perspectives and recent advances in major animal models of hypertension. Acta Pharmacol Sin. 2005;26: 295-301.

3 Lisowska-Myjak B. Serum and urinary biomarkers of acute kidney injury. Blood Purif. 2010;29:357-65.

4 Quesada A, Vargas F, Montoro-Molina S, O’Valle F, Rodríguez-Martínez MD, Osuna A, et al. Urinary aminopeptidase activities as early and predictive biomarkers of renal dysfunction in cisplatin-treated rats. PLoS One. 2012;7(7):e40402.

5 Montoro-Molina S, Quesada A, Zafra-Ruiz PV, O’Valle F, Vargas F, de Gracia Mdel C, et al. Immunological detection of glutamyl aminopeptidase in urine samples from cisplatintreated rats. Proteomics Clin Appl. 2015;9: $630-5$.
6 Pérez-Abud R, Rodríguez-Gómez I, Villarejo $\mathrm{AB}$, Moreno JM, Wangensteen R, Tassi M, et al. Salt sensitivity in experimental thyroid disorders in rats. Am J Physiol Endocrinol Metab. 2011;301:E281-7.

7 Montoro-Molina S, López-Carmona A, Quesada A, O’Valle F, Martín-Morales N, Osuna $\mathrm{A}$, et al. Klotho and aminopeptidases as early biomarkers of renal injury in Zucker obese rats. Front Physiol. 2018;9:1599.

8 Kuro-o M, Matsumura Y, Aizawa H, Kawaguchi $\mathrm{H}$, Suga T, Utsugi T, et al. Mutation of the mouse klotho gene leads to a syndrome resembling ageing. Nature. 1997;390:45-51.

9 Ito S, Kinoshita S, Shiraishi N, Nakagawa S, Sekine S, Fujimori T, et al. Molecular cloning and expression analyses of mouse betaklotho, which encodes a novel Klotho family protein. Mech Dev. 2000;98:115-9.

$10 \mathrm{Hu}$ MC, Shi M, Zhang J, Addo T, Cho HJ, Barker SL, et al. Renal production, uptake, and handling of circulating aKlotho. J Am Soc Nephrol. 2016;27:79-90.

11 Wang Y, Sun Z. Klotho gene delivery prevents the progression of spontaneous hypertension and renal damage. Hypertension. 2009;54: $810-7$.
12 Cheng X, Zhou Q, Lin S, Wu R. Fosinopril and valsartan intervention in gene expression of Klotho, MMP-9, TIMP-1, and PAI-1 in the kidney of spontaneously hypertensive rats. Zhong Nan Da Xue Xue Bao Yi Xue Ban. 2010;35:1048-56.

13 Tang R, Zhou Q, Liu Z, Xiao Z, Pouranan V. Effects of fosinopril and losartan on renal Klotho expression and oxidative stress in spontaneously hypertensive rats. Zhong Nan Da Xue Xue Bao Yi Xue Ban. 2011;36:27-33.

14 Wang Y, Sun Z. Antiaging gene Klotho regulates endothelin-1 levels and endothelin receptor subtype B expression in kidneys of spontaneously hypertensive rats. J Hypertens. 2014;32:1629-36.

15 Shimamura Y, Hamada K, Inoue K, Ogata K, Ishihara M, Kagawa T, et al. Serum levels of soluble secreted $\alpha$-Klotho are decreased in the early stages of chronic kidney disease, making it a probable novel biomarker for early diagnosis. Clin Exp Nephrol. 2012;16:722-9.

16 Scholze A, Liu Y, Pedersen L, Xia S, Roth HJ, Hocher B, et al. Soluble $\alpha$-klotho and its relation to kidney function and fibroblast growth factor-23. J Clin Endocrinol Metab. 2014;99: E855-61. 
17 Lambeir AM, Durinx C, Scharpé S, De Meester I. Dipeptidyl-peptidase IV from bench to bedside: an update on structural properties, functions, and clinical aspects of the enzyme DPP IV. Crit Rev Clin Lab Sci. 2003;40:209-94.

18 Kenny AJ, Booth AG, George SG, Ingram J, Kershaw D, Wood EJ, et al. Dipeptidyl peptidase IV, a kidney brush-border serine peptidase. Biochem J. 1976;157:169-82.

19 Iwaki-Egawa S, Watanabe Y, Kikuya Y, Fujimoto Y. Dipeptidyl peptidase IV from human serum: purification, characterization, and $\mathrm{N}$ terminal amino acid sequence. J Biochem. 1998; 124:428-33.

20 Nistala R, Savin V. Diabetes, hypertension, and chronic kidney disease progression: role of DPP4. Am J Physiol Renal Physiol. 2017; 312:F661-70

21 Zhong J, Rao X, Rajagopalan S. An emerging role of dipeptidyl peptidase 4 (DPP-4) beyond glucose control: potential implications in cardiovascular disease. Atherosclerosis. 2013; 226:305-14.

22 Shih W, Hines WH, Neilson EG. Effects of cyclosporin A on the development of immunemediated interstitial nephritis. Kidney Int. 1988;33:1113-8.

23 Sweat F, Puchtler H, Rosenthal SI. Sirius red F3BA as a stain for connective tissue. Arch Pathol. 1964;78:69-72.

24 Hultström M. Development of structural kidney damage in spontaneously hypertensive rats. J Hypertens. 2012;30:1087-91.

25 Dworkin LD, Feiner HD. Glomerular injury in uninephrectomized spontaneously hypertensive rats. A consequence of glomerular capillary hypertension. J Clin Invest. 1986;77: 797-809.
26 Feld LG, Van Liew JB, Galaske RG, Boylan JW. Selectivity of renal injury and proteinuria in the spontaneously hypertensive rat. Kidney Int. 1977; 12:332-43.

27 Bakoush O, Tencer J, Torffvit O, Tenstad O, Skogvall I, Rippe B. Increased glomerular albumin permeability in old spontaneously hypertensive rats. Nephrol Dial Transplant. 2004;19:1724-31.

28 Hultstrom M, Leh S, Skogstrand T, Iversen BM. Upregulation of tissue inhibitor of metalloproteases-1 (TIMP-1) and procollagen-Npeptidase in hypertension-induced renal damage. Nephrol Dial Transplant. 2008;23: 896-903.

29 Gilbert RE. Proximal tubulopathy: prime mover and key therapeutic target in diabetic kidney disease. Diabetes. 2017;66(4):791800.

30 Reckelhoff JF, Zhang H, Granger JP. Decline in renal hemodynamic function in aging SHR: role of androgens. Hypertension. 1997;30(Pt 2):677-81.

31 Chamorro V, Wangensteen R, Sainz J, Duarte J, O'Valle F, Osuna A, et al. Protective effects of angiotensin II type 1 (AT1) receptor blockade in low renin deoxicorticosterone acetate (DOCA)-treated spontaneously hypertensive rats. Clin Sci. 2004;106:1-9.

32 Kobori H, Nishiyama A, Harrison-Bernard LM, Navar LG. Urinary angiotensinogen as an indicator of intrarenal angiotensin status in hypertension. Hypertension. 2003;41:429.

33 Quesada A, Segarra AB, Montoro-Molina S, de Gracia MD, Osuna A, O'Valle F, et al. Glutamyl aminopeptidase in microvesicular and exosomal fractions of urine is related with renal dysfunction in cisplatin-treated rats. PLoS One. 2017;12(4):e0175462.
34 Song L, Ye M, Troyanovskaya M, Wilk E, Wilk S, Healy DP. Rat kidney glutamyl aminopeptidase (aminopeptidase A): molecular identity and cellular localization. Am J Physiol. 1994;267:F546-57.

35 Pacheco BP, Crajoinas RO, Couto GK, Davel AP, Lessa LM, Rossoni LV, et al. Dipeptidyl peptidase IV inhibition attenuates blood pressure rising in young spontaneously hypertensive rats. J Hypertens. 2011;29:520-8.

36 Liu L, Liu J, Wong WT, Tian XY, Lau CW, Wang YX, et al. Dipeptidyl peptidase 4 inhibitor sitagliptin protects endothelial function in hypertension through a glucagon-like peptide 1-dependent mechanism. Hypertension. 2012;60(3):833-41

37 Cho EH, Kim SW. Soluble dipeptidyl peptidase- 4 levels are associated with decreased renal function in patients with type 2 diabetes mellitus. Diabetes Metab J. 2019;43:97-104.

38 Yang G, Li Y, Cui L, Jiang H, Li X, Jin C, et al. Increased plasma dipeptidyl peptidase- 4 activities in patients with coronary artery disease. PLoS One. 2016;11:e0163027.

39 Duvnjak L, Blaslov K, Vučić Lovrenčić M, Knežević Ćuća J. Persons with latent autoimmune diabetes in adults express higher dipeptidyl peptidase- 4 activity compared to persons with type 2 and type 1 diabetes. Diabetes Res Clin Pract. 2016;121:119-26.

40 Yazbeck R, Jaenisch SE, Abbott CA. Potential disease biomarkers: dipeptidyl peptidase 4 and fibroblast activation protein. Protoplasma. 2017;23:1-12.

41 Hirose M, Takano H, Hasegawa H, Tadokoro $\mathrm{H}$, Hashimoto N, Takemura G, et al. The effects of dipeptidyl peptidase- 4 on cardiac fibrosis in pressure overload-induced heart failure. J Pharmacol Sci. 2017;135:164-73. 